Jitan Lu and Peng-Yee Lee, Division of Mathematics, School of Science, National Institute of Education, 469 Bukit Timah Road, Singapore, 259756.

e-mail: lujitan@hotmail.com and pylee@nie.edu.sg

\title{
ON SINGULARITY OF HENSTOCK INTEGRABLE FUNCTIONS
}

\begin{abstract}
We define a singular point of a Henstock integrable function to be one which is not contained in any open interval on which the function is Lebesgue integrable. Then we give examples to illustrate the possible measure of the set of such singular points.
\end{abstract}

\section{Introduction}

It is well known that for any Henstock integrable function defined on a nondegenerate closed bounded interval $I$ on the real line, there exists a nondegenerate subinterval on which it is Lebesgue integrable [1]. Then we can obtain a set which is the union of all subintervals on each of which the function is Lebesgue integrable. A question arises naturally: can we give an estimate of the measure of the union? To see this question clearly, we introduce a new concept. Let $f$ be a Henstock integrable function on a nondegenerate closed bounded interval $I$ on the real line. A point $x \in I$ is called regular if there exists an open interval $I(x)$ containing $x$, such that $f$ is Lebesgue integrable on $I \cap I(x)$. We denote the set of all regular points in $I$ by $W(f)$ and $U(f)=I \backslash W(f)$. We call the points in $U(f)$ singular points. It is obvious that $U(f)=\emptyset$ if and only if $f$ is Lebesgue integrable on $I$. Thus the singular points are the essential difference of a Henstock integrable function from a Lebesgue integrable function and the existence of singular points determines that a Henstock function is non-absolutely integrable. Moreover, $W(f)$ is the union of all the open subintervals on each of which $f$ is Lebesgue integrable. Thus the above question is equivalent to estimating the possible measure of $U(f)$. In this short paper, we shall give some examples to illustrate the possible $U(f)$ for a Henstock integrable function.

Key Words: Singular point; Henstock integrable function; Lebesgue integrable function

Mathematical Reviews subject classification: 26A39

Received by the editors February 12, 1998 


\section{The Singularity of Henstock Integrable Functions}

Let $f$ be a Henstock integrable function on a nondegenerate closed bounded interval $I$ on the real line. Since $W(f)$ is an non-empty open set [1], $U(f)$ is a closed set which measure is less than the measure of $I$. Consider the function $f(x)=\frac{1}{x} \sin \frac{1}{x}$ when $0<x \leq 1$ and 0 when $x=0$. In this well-known example, $f$ is Henstock integrable on $[0,1]$ and is Lebesgue integrable on $[a, 1]$ for any $a \in(0,1]$. Thus $U(f)$ is a single point set $\{0\}$. Based on this example, it is easy to construct examples with $U(f)$ being a finite or countable set. On the other hand, after checking many well-known Henstock functions, we find that $U(f)$ is always a very "small" set. So we conjecture that the $U(f)$ is always a measure zero set. But it is not true. In fact $U(f)$ can be a positive measure set. In the following, we shall give a further example.

Let $[a, b]$ be a nondegenerate closed bounded interval. We define a function $\varphi_{[a, b]}$ on $[a, b]$ as follows. Let $\Psi_{[a, b]}(x)=(x-a)^{2}(x-b)^{2} \sin \frac{1}{(x-a)^{2}(x-b)^{2}}$ when $x \in(a, b)$ and $\Psi_{[a, b]}(x)=0$ when $x=a$ or $b$. It is obvious that $\Psi_{[a, b]}$ is differentiable on $[a, b]$. Denote its derivative by $\varphi_{[a, b]}(x)$. It is easy to check that $\varphi_{[a, b]}$ is Henstock integrable but not Lebesgue integrable on $[a, b]$. Moreover, $U\left(\varphi_{[a, b]}\right)=\{a, b\}$. Next we construct a Henstock integrable function $f$ on the interval $[0,1]$ from the above function $\varphi_{[a, b]}$. First, on $[0,1]$, we construct a generalized Cantor set $X$ of measure $\frac{1}{2}[2, \mathrm{p} .41]$ with the complementary open intervals given by $J_{i, j}^{\circ}, i=1,2, \ldots, j=1, \ldots, 2^{i-1}$, in which the measure of $J_{i, j}$ is $\frac{1}{2^{i-1} 3^{i}}$, where $J_{i, j}^{\circ}$ denotes the interior of the interval $J_{i, j}$. Now we define two functions on $[0,1]$ as follows:

$$
f(x)= \begin{cases}0 & \text { when } x \in X \\ \varphi_{J_{i, j}}(x) & \text { when } x \in J_{i, j}^{\circ}, i=1,2, \ldots, j=1, \ldots, 2^{i-1},\end{cases}
$$

and

$$
F(x)= \begin{cases}0 & \text { when } x \in X \\ \Psi_{J_{i, j}}(x) & \text { when } x \in J_{i, j}^{\circ}, i=1,2, \ldots, j=1, \ldots, 2^{i-1} .\end{cases}
$$

We shall prove that $F$ is differentiable and $F^{\prime}(x)=f(x)$ for every $x \in[0,1]$ and then $f$ is Henstock integrable on $[0,1]$. The case when $x \in J_{i, j}^{\circ}, i=1,2, \ldots, j=$ $1, \ldots 2^{i-1}$ is obvious. Next, let $x$ be an endpoint of certain $J_{i, j}$. Suppose that it is the right endpoint, we can easily see that the left derivative of $F$ at $x$ exists and equals to zero. On the other hand, for any $\varepsilon$ with $1>\varepsilon>0$, let $x_{1} \in(x, x+\varepsilon)$ be the right end point of certain $J_{s, t}$. If $y \in X \cap\left[x, x_{1}\right]$, then $F(y)=0$. If $y \in J_{k, l}^{\circ} \subset\left[x, x_{1}\right]$ for some $J_{k, l}=\left[x_{0}, y_{0}\right]$, then

$$
\left|\frac{F(y)-F(x)}{y-x}\right| \leq\left|\frac{\Psi_{J_{k, l}}(y)}{y-x_{0}}\right| \leq\left(y_{0}-x_{0}\right)^{3}<\varepsilon .
$$


Thus, the right derivative of $F$ at $x$ exists and equals to zero. The proof for the left endpoint of $J_{i, j}$ is similar. Finally, for $x \in[0,1] \backslash \cup_{i, j} J_{i, j}$, following the same argument above, we can prove that both of the right and the left derivatives of $F$ at $x$ exist and equal to zero. Now we shall prove that $U(f)=X$ for the Henstock integrable function $f$. Let $x \in U(f)$. Then $x \notin \cup_{i, j} J_{i, j}^{\circ}$, that is $x \in X$. On the other hand, let $x$ be any point in $X$. If $x$ is one of the end points of certain $J_{i, j}$, it is obvious that $x \in U(f)$. If $x \in[0,1] \backslash \cup_{i, j} J_{i, j}$ and suppose $x \notin U(f)$, then there exists an open interval $I$ containing $x$ on which $f$ is Lebesgue integrable. But we know that there exists some interval $J_{s, t} \subset I$ and $f$ is not Lebesgue integrable on it. This is a contradiction. Therefore $x \in U(f)$ and then $U(f)=X$. We have constructed above a Henstock integrable function $f$ on $[0,1]$ with the measure of the set of singular points being $\frac{1}{2}$. In fact, nin a similar manner, we can construct a Henstock integrable function with the measure of the set of singular points being $\lambda$ on interval $[0,1]$ for any $0<\lambda<1$. This means that there are Henstock integrable functions on an interval that admit a set of singular points with its measure as close as possible but not equal to that of the whole interval.

\section{References}

[1] Z. Buczolich, Henstock integrable functions are Lebesgue integrable on a portion, Proc. Amer. Math. Soc., 111(1991), 127-129.

[2] G. B. Folland, Real analysis, modern techniques and their applications, New York, 1984.

[3] P. Y. Lee, Lanzhou lectures on Henstock integral, World Scientific, 1989.

[4] S. Saks, Theory of the integral, New York, 1937. 
